\title{
Mortalidade Materna na Cidade do Recife
}

\author{
Maternal Mortality in Recife
}

Aurélio Antônio Ribeiro Costa, Maria do Socorro Sampaio de Sousa Ribas, Melania Maria Ramos de Amorim, Luiz Carlos Santos

\begin{abstract}
RESUMO
Objetivos: determinar a Razão de Mortalidade Materna (RMM) entre mulheres residentes na cidade do Recife, pela análise de todas as declarações de óbito de mulheres na idade entre 10-49 anos. Determinar a taxa de sub-registro e estudar as principais caracteristicas, causas básicas, classificação e evitabilidade das mortes maternas.

Métodos: realizou-se estudo descritivo de base populacional, analisando-se todos as declarações de óbito de mulheres entre 10-40 anos e utilizando-se os critérios de Laurenti para classificá-los como declarados ou presumiveis. Estudaram-se os prontuários médicos e os dados de autópsia, quando disponiveis, determinando-se as causas básicas dos óbitos e calculando-se a taxa de sub-registro. A Razão de Mortalidade Materna foi calculada usando as informações sobre nascidos vivos do SINASC (Sistema de Informações dos Nascidos Vivos). Resultados: encontraram-se 144 mortes maternas, sendo 104 declaradas e 44 presumiveis, confirmadas após investigação. A Razão de Mortalidade Materna foi 75,5 por 100.000 nascidos vivos e o percentual de sub-registro foi 27,8\%. Observou-se uma predominância de causas diretas, sendo as mais freqüentes hipertensão (19\%), hemorragia (16\%) e infecção (11\%). Cerca de $82 \%$ das mortes foram consideradas evitáveis por meio de assistência adequada ao pré-natal, parto e puerpério.

Conclusões: a Razão de Mortalidade Materna é alta na cidade de Recife, e o percentual de subnotificação permanece elevado. Predominam as causas diretas e os óbitos evitáveis, evidenciando ausência de assistência adequada ao pré-natal, parto e puerpério.
\end{abstract}

PALAVRAS-CHAVE: Mortalidade materna. Mortalidade reprodutiva. Causas de morte. Estatísticas vitais.

\section{Introdução}

A definição clássica de morte materna considera "a morte de uma mulher durante a gestação ou dentro de um período de 42 dias após o término da gestação, independentemente da duração ou da localização da gravidez, devida a qualquer causa relacionada com ou agravada pela gestação ou por medidas tomadas em relação a ela, porém não devidas a causas acidentais ou incidentais" ${ }^{1}$.

Essa definição tem sido amplamente ques-

Instituto Materno-Infantil de Pernambuco (IMIP)

Correspondência:

Melania Amorim

Coordenação de Ensino do IMIP

Rua dos Coelhos, 300 - Ilha do Leite

50050-570 - Recife - PE

e-mail: melamorim@uol.com.br tionada nos últimos anos, uma vez que o progressivo avanço tecnológico (por exemplo, técnicas de respiração assistida) tem freqüentes vezes conseguido prolongar a vida além desses 42 dias, ocorrendo por exemplo dois, três meses após o parto a morte de uma mulher cuja causa básica esteve diretamente relacionada ao ciclo gestatório.

A FIGO recomenda que sejam consideradas como mortes maternas "todas as mortes, independentemente da causa, que ocorram até 42 dias após o término da gestação, e todas as mortes em que tenha sido possivel identificar como desencadeante da causa o processo gestacional, até um ano pós-parto". As mortes não obstétricas, portanto, deveriam fazer parte dos cálculos da mortalidade materna, incluindo assim as causas acidentais e incidentais, como por exemplo os homicídios, suicídios e mortes por trauma, que em 
algumas regiões representam mesmo a causa mais freqüente de morte materna ${ }^{2}$.

O conceito de morte materna tardia foi introduzido pela OMS pela $10^{a}$ Revisão da CID (1994), incorporando parcialmente as recomendações da FIGO e passando a incluir "a morte de uma mulher por causas obstétricas diretas ou indiretas ocorridas entre 42 dias até um ano depois do término da gravidez"3.

As mortes maternas obstétricas podem ser classificadas como diretas ou indiretas. As mortes diretas resultam de complicações obstétricas relacionadas à gravidez, parto e puerpério, devidas a intervenções, omissões, tratamento incorreto ou de uma seqüência de eventos resultantes de qualquer uma dessas situações (ex.: hemorragia, infecção puerperal, hipertensão, tromboembolismo, acidente anestésico). Representam ainda a principal causa de morte materna nos países em desenvolvimento. As mortes indiretas decorrem de doenças preexistentes ou que se desenvolvem durante a gestação (intercorrentes) e que não se devem a causas obstétricas diretas, mas que foram agravadas pelos efeitos fisiológicos da gestação (ex.: cardiopatias, colagenoses e outras doenças crônicas).

A mortalidade materna representa grave problema de saúde pública. Estima-se que 600.000 mulheres morram anualmente no mundo durante o ciclo gravídico-puerperal (uma morte materna por minuto), sendo que $99 \%$ dessas mortes ocorrem nos países em desenvolvimento. Esses países são responsáveis por $86 \%$ dos nascimentos em todo o mundo ${ }^{4}$.

Há várias décadas já se dispõe do conhecimento e dos meios necessários para remover o risco relacionado à concepção, de forma que nenhuma mulher deveria morrer por complicações associadas à gravidez, parto e puerpério. Em sua quase totalidade, as mortes maternas são evitáveis por uma boa assistência pré-natal, perinatal (parto) e pós-natal imediata (puerpério).

Em países como Finlândia, Suécia, Holanda, Canadá, Noruega e outros, a mortalidade materna encontra-se em torno de 4 para 100.000 nascidos vivos, admitindo-se mesmo que se possa alcançar situação em que não mais ocorram mortes maternas. A OMS considera aceitável uma razão de morte materna entre 10 e 20 por 100.000 nascidos vivos ${ }^{4}$.

No entanto, em várias regiões do mundo, a mortalidade materna continua sendo bastante alta. Em países africanos como Nigéria, Lesotho, Somália e outros, têm sido descritas taxas de até 1000 mortes por 100.000 nascidos vivos (algo em torno de uma morte materna em cada 100 nascimentos!!!). Na América Latina, calcula-se haver
270 óbitos maternos por 100.000 nascidos vivos; na Ásia, 420 por 100.000 nascidos vivos. A morte materna chega a constituir cerca de $20-45 \%$ dos óbitos femininos em idade fértil nos países em desenvolvimento e ao redor de $1 \%$ nos países desenvolvidos ${ }^{4}$.

A mortalidade materna constitui retrato fiel do painel sócio-político-cultural da sociedade, da mesma maneira que o coeficiente de mortalidade infantil constitui indicador fiel do nivel de saúde da população, relacionando-se à disponibilidade $\mathrm{e}$ qualidade dos recursos de saúde existentes.

De acordo com a Organização Mundial de Saúde (OMS) e o "United Nations International Children's Emergency Fund" (UNICEF), "a morte materna representa um indicador do status da mulher, seu acesso à assistência à saúde e a adequação do sistema de assistência à saúde em responder às suas necessidades. É preciso, portanto, ter informações sobre niveis e tendências da mortalidade materna, não somente pelo que ela estima sobre os riscos na gravidez e no parto mas também pelo que significa sobre a saúde em geral da mulher e, por extensão, seu status social e econômico" ${ }^{4}$.

Estimar a magnitude do problema é um dos primeiros passos para identificar suas causas e deliberar estratégias para reduzir a mortalidade materna. No entanto, embora as estatísticas oficiais já tão elevadas sejam um alerta, o número real de mortes maternas permanece subestimado. Por meio de informações adicionais, vários estudos têm demonstrado a precariedade da informação sobre a mortalidade; e, embora a subenumeração ocorra mesmo em países desenvolvidos, com bom sistema de registro (estudos realizados nos Estados Unidos evidenciam subestimativa de $25-40 \%)^{2}$, é nos países em desenvolvimento, com taxas mais altas, que é maior o sub-registro; na Jamaica, por exemplo, chega a $100 \%{ }^{4}$.

No Brasil, diversos estudos têm evidenciado elevado sub-registro dos óbitos maternos, observando-se pela investigação de todos os óbitos femininos em idade fértil um sub-registro em torno de $40 \%{ }^{5}$. Em vários casos, os registros de óbitos não mencionam a presença de gravidez, e o diagnóstico de causa básica da morte pode ocultar a causa real, associada à reprodução. Assim, o diagnóstico de "falência de múltiplos órgãos" em conseqüência de uma septicemia muitas vezes mascara uma morte decorrente do abortamento ilegal, provocado sem as minimas condições de anti-sepsia.

Assim, embora as estatísticas oficiais do Ministério da Saúde (1980-1997) apontem para uma razão de mortalidade materna de 50/100.000 nascidos vivos ${ }^{6}$, os estudos de Laurenti demonstram 
a necessidade de se aplicar um fator de correção de 2.2 (dada a magnitude do sub-registro) ${ }^{7,8}$. O Ministério da Saúde trabalha portanto com a taxa corrigida de 110 óbitos/100.000 nascidos vivos para o mesmo período. Essa mortalidade é extremamente elevada em relação aos países desenvolvidos, destacando-se outrossim que grande parte dos óbitos devem-se a causas diretas e, em sua maioria, evitáveis ${ }^{9}$.

O presente estudo teve por objetivo determinar a Razão de Mortalidade Materna para as mulheres residentes na cidade do Recife, analisando a magnitude do sub-registro pela avaliação de todas as declarações de óbito em mulheres entre 10 e 49 anos. Estudaram-se ainda as principais características, classificação e evitabilidade das mortes maternas.

\section{Métodos}

Realizou-se estudo descritivo, de base populacional, analisando-se todas as declarações de óbito de mulheres na faixa etária entre 10-49 anos residentes na cidade do Recife, entre 1994 e 2000. A partir destas, identificaram-se as mortes maternas tanto declaradas como presumiveis, realizando-se visitas domiciliares com entrevista dos familiares, investigando-se os registros hospitalares, prontuários médicos e dados de necropsia, quando disponiveis. Todas as informações pertinentes foram coletadas pela Secretaria de Saúde da cidade do Recife.

Definiu-se como morte materna "a morte de uma mulher durante a gestação ou dentro de um periodo de 42 dias após o término da gestação, independentemente da duração ou da localização da gravidez, devida a qualquer causa relacionada com ou agravada pela gestação ou por medidas tomadas em relação a ela, porém não devidas a causas acidentais ou incidentais". As mortes com mais de 42 dias e até um ano depois do término da gravidez foram consideradas como "morte materna tardia"

Adotaram-se os critérios preconizados por Laurenti para diferenciar, entre as declarações de óbito, as mortes maternas declaradas, presumiveis e aquelas não relacionadas a gravidez, parto e puerpério ${ }^{7}$.

Mortes declaradas foram aquelas em que se especificava claramente o estado gravídicopuerperal na causa descrita na declaração de óbito ou se fazia menção ao estado gestacional nos campos 43, 44 e 49 no bloco VI da declaração de óbito adotada em 1999. Consideraram-se mortes não declaradas quando na declaração de óbito não constava nenhuma referência de gravidez, parto ou puerpério, e após investigação do prontuário, visitas domiciliares, necropsia ou outro meio, ficou evidente o estado gravídico-puerperal.

Excluíram-se as mortes maternas não obstétricas, ou seja, aquelas resultantes de causas acidentais ou incidentais, não relacionadas com a gravidez ou sua condução, que não entram para o cálculo da Razão de Mortalidade Materna.

Para cálculo da razão de mortalidade materna (RMM), utilizaram-se os dados do SINASC (Sistema de Informações dos Nascidos Vivos) referentes a cada ano estudado para toda a cidade do Recife (disponibilizados os dados pela Secretaria de Saúde), obedecendo-se à fórmula padrão:

$\mathrm{RMM}=\mathrm{n}^{\circ}$ de óbitos obstétricos diretos e indiretos / $\mathrm{n}^{\circ}$ de nascidos vivos $\mathrm{x} 100.000$

Analisaram-se as seguintes variáveis: classificação da morte materna (direta ou indireta) e causas básicas do óbito, local de ocorrência do óbito (hospital, transporte, domicílio ou via pública), transferência hospitalar, período do óbito (gravidez, parto ou pós-parto), intervalo (dias) entre parto/aborto e o óbito, características maternas (idade, cor, escolaridade, número de gestações e partos, idade gestacional), dados pré-natais (assistência pré-natal e número de consultas), local do parto, tipo de parto (transvaginal espontâneo, a fórcipe ou cesárea) e avaliação do óbito como evitável (incluindo as medidas preventivas cabiveis) ou inevitável.

Considerou-se como morte obstétrica direta aquela decorrente de complicações obstétricas relacionadas à gravidez, parto e puerpério, devidas a intervenções, omissões, tratamento incorreto ou de uma seqüência de eventos resultantes de qualquer uma dessas situações. Por outro lado, classificou-se a morte obstétrica como indireta quando decorrente de doenças preexistentes ou que se desenvolvem durante a gestação (intercorrentes), não se devendo a causas obstétricas diretas, mas que foram agravadas pelos efeitos fisiológicos da gestação ${ }^{3}$.

A determinação da causa básica de óbito foi realizada caso a caso, após a investigação, considerando-se a Classificação Internacional de Doenças $\left(10^{a}\right.$ revisão) para mortes obstétricas diretas (códigos $\mathrm{O} 00$ a $\mathrm{O} 08$ e $\mathrm{O} 10$ a O92), indiretas (códigos 098 a O99) e de causa desconhecida (código O95) ${ }^{3}$.

Todos os dados foram digitados em arquivo específico criado no programa Epi-Info 2000 para Windows, gerando-se assim um banco de dados que foi posteriormente utilizado para análise estatística. Para análise descritiva, utilizaram-se distribuições de freqüência para as variáveis categóri- 
cas e medidas de tendência central (média e mediana) e de dispersão (desvio-padrão) para as variáveis numéricas.

O presente estudo respeitou as normas da Declaração de Helsinque e da resolução no 196/ 96 do Conselho Nacional de Saúde para pesquisa em seres humanos, tendo sido aprovado pelo Comitê de Ética em Pesquisa do IMIP.

\section{Resultados}

Ocorreram 4611 mortes de mulheres com ida- de de 10 a 49 anos entre 1994 e 2000 na cidade do Recife. Destas, 144 foram mortes maternas, sendo 104 declaradas nos atestados de óbito e 40 confirmadas após investigação dos óbitos presumiveis. A freqüência de sub-registro foi de $27,8 \%$, observando-se uma tendência ao declínio da subnotificação nos últimos dois anos (1999 e 2000) (Tabela 1).

Considerando o total de nascidos vivos entre 1994 e 2000 (190.684), determinou-se a Razão de Mortalidade Materna (RMM) para esse periodo, obtendo-se o valor de 75,5 por 100.000 . A RMM foi próxima de 100 nos anos de 1994 e 1997, encontrando-se os valores mais baixos nos anos de 1995 e 1966 (abaixo de 60), estabilizando-se em torno

Tabela 1 - Distribuição de freqüência dos casos de morte feminina entre 10-49 anos e morte materna na cidade do Recife (1994-2000), de acordo com sua classificação (declarada ou presumivel) e magnitude do sub-registro

\begin{tabular}{lccccccr}
\hline Ano & $\begin{array}{c}\text { Mortes femininas } \\
\text { 10-49 anos }\end{array}$ & Declaradas & $\begin{array}{c}\text { Mortes maternas } \\
\text { Não } \\
\text { declaradas }\end{array}$ & $\begin{array}{c}\text { Total de mortes } \\
\text { maternas }\end{array}$ & \% sub-registro & No de nascidos vivos & RMM \\
\hline 1994 & 628 & 17 & 10 & 27 & 37,0 & 28.429 & 94,97 \\
1995 & 676 & 14 & 02 & 16 & 12,5 & 28.079 & 56,98 \\
1996 & 658 & 08 & 08 & 16 & 50,0 & 27.540 & 58,10 \\
1997 & 689 & 20 & 07 & 27 & 25,9 & 27.197 & 99,28 \\
1998 & 694 & 16 & 07 & 23 & 30,4 & 26.512 & 86,75 \\
1999 & 644 & 15 & 03 & 18 & 16,7 & 27.584 & 65,25 \\
2000 & 661 & 14 & 03 & 17 & 17,6 & 25.343 & 67,08 \\
TOTAL & 4611 & 104 & 40 & 144 & 27,8 & 190.684 & 75,52 \\
\hline
\end{tabular}

Fonte: Secretaria de Saúde do Recife

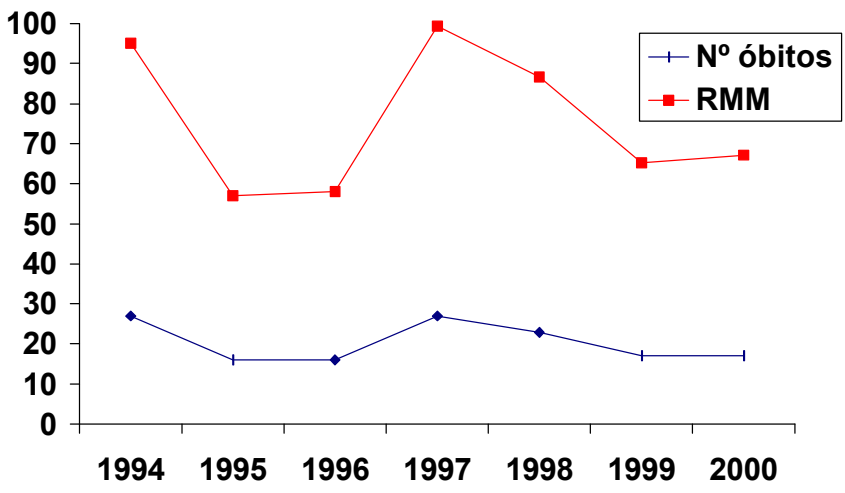

Figura 1 - Número de óbitos maternos e Razão de Mortalidade Materna na cidade do Recife (1994-2000)

de 65 nos anos de 1999 e 2000 (Figura 1).

Verificou-se nítido predomínio das causas obstétricas diretas (quase $70 \%$ ) de morte materna, sendo as causas mais freqüentes hipertensão $(19,4 \%)$, hemorragia $(16 \%)$ e infecção puerperal (11\%). Mortes decorrentes de complicações de abortamento e prenhez ectópica representaram respectivamente 9,0 e 5,0\% dos casos. Por outro lado, entre as causas indiretas, destacaram-se as cardio-

Tabela 2 - Distribuição de freqüência dos óbitos maternos na cidade do Recife (19942000) de acordo com a classificação e a causa básica

\begin{tabular}{lcc}
\hline Causa Básica & Freqüência Absoluta & $\%$ \\
\hline Mortes Obstétricas Diretas & 99 & 68,8 \\
Hipertensão & 28 & 19,4 \\
Hemorragia & 23 & 16,0 \\
Infecção puerperal & 16 & 11,1 \\
Complicações de abortamento & 13 & 9,0 \\
Prenhez ectópica & 8 & 5,5 \\
Embolia pulmonar & 6 & 4,2 \\
Outras causas diretas & 5 & 3,5 \\
Mortes Obstétricas Indiretas & 45 & 31,2 \\
Cardiopatia & 18 & 12,5 \\
Pneumonia & 11 & 7,6 \\
AVC/hemorragia cerebral & 6 & 4,2 \\
Outras infecções & 5 & 3,5 \\
Outras causas indiretas & 5 & 3,5 \\
Total & 144 & 100,0 \\
\hline Fonte: Secretaria de Saúde do Recife & &
\end{tabular}


patias como as mais freqüentes (12,5\%) (Tabela 2).

A maioria dos óbitos ocorreu em hospital (mais de 90\%), porém verificaram-se óbitos durante o transporte ou no domicílio $(4,2$ e $3,5 \%$, respectivamente). Quase $80 \%$ dos óbitos ocorreram em hospitais públicos, filantrópicos ou universitários. Encontrou-se uma freqüência de transferência hospitalar de 38,5\%.

A morte ocorreu em grande parte dos casos durante o puerpério (em torno de 67\%), e apenas $1,4 \%$ dos casos ocorreram durante o parto. Vinte e cinco casos $(17,4 \%)$ aconteceram durante a gravidez. Considerando-se as mortes no puerpério, 98 (em torno de $68 \%$ do total) ocorreram até 42 dias depois do parto e 9 (6,3\% do total) com mais de 42 dias transcorridos desde o parto.

A idade materna variou entre 14 e 43 anos (média de 27,7 anos), observando-se a Razão de Mortalidade Materna (RMM) mais elevada na faixa etária a partir dos 35 anos (237 por 100.000 nascidos vivos). Praticamente não houve diferença entre as adolescentes (até 19 anos) e as mulheres entre 20-34 anos, com RMM em torno de 65 por 100.000 nascidos vivos.

Em relação às características maternas, encontrou-se freqüência de $37 \%$ de mulheres da cor parda, porém a informação sobre cor não foi obtida em 38\% dos casos, prejudicando assim a análise. Também não foram obtidas informações sobre a escolaridade de mais de $50 \%$ dos casos, encontrando-se em torno de $4 \%$ de nivel universitário, $15 \%$ de segundo grau, $25 \%$ de primeiro grau e $3,5 \%$ de pacientes sem nenhuma escolaridade. A paridade variou entre 0 e 11 , com mediana de 1 , sendo que a maioria das mulheres já tinham 1-3 partos anteriores (aproximadamente 55\%). A idade gestacional variou entre 4 e 42 semanas (mediana de 35), encontrando-se o maior percentual de óbitos no terceiro trimestre (68\%).

Encontrou-se um percentual de $22 \%$ de mulheres que não receberam qualquer cuidado pré-natal, porém informações sobre o pré-natal não foram obtidas em $25 \%$ dos casos. A informação sobre o número de consultas também foi falha (informação ausente em quase $60 \%$ dos casos). Esse número variou entre 1-10 consultas (mediana de 5), com um percentual de $18 \%$ de 6 ou mais consultas. A maioria dos partos (em torno de 66\%) foram assistidos em hospitais públicos, filantrópicos ou universitários.

Analisando-se o tipo de parto, observou-se uma freqüência mais elevada de operação cesariana (65\%), com uma Razão de Mortalidade Materna próxima de 85 por 100.000 , ao passo que a RMM para parto vaginal foi de 29,5 por 100.000 nascidos vivos.

A maior freqüência de mortes maternas foi verificada nos domingos (em torno de $20 \%$ ), seguindo-se os sábados (16\%) e as terças e quintas (14,6\% cada). A menor freqüência foi encontrada nas quartas-feiras (em torno de 10\%). Quase 32\% dos óbitos ocorreram na madrugada (entre 0 e 6 h59min). Em relação aos meses do ano, a freqüência de óbitos foi maior nos meses de dezembro $(12,5 \%)$ e junho $(11,8 \%)$.

Consideraram-se evitáveis $82 \%$ das mortes maternas (118 casos), por meio de medidas educativas e assistência adequada ao pré-natal, ao parto e ao puerpério. Dezessete óbitos $(11,8 \%)$ foram considerados inevitáveis (Tabela 3 ).

Tabela 3 - Distribuição de freqüência dos óbitos maternos de acordo com sua evitabilidade e medidas preventivas cabíveis

\begin{tabular}{lcc}
\hline Evitabilidade/Medidas preventivas & Freqüência Absoluta & $\%$ \\
\hline Evitáveis & 118 & 81,9 \\
Medidas educativas & 59 & 41,0 \\
Assistência pré-natal adequada & 60 & 41,7 \\
Melhor acesso ao sistema de saúde & 19 & 13,2 \\
Assistência adequada ao parto & 45 & 31,3 \\
Assistência adequada ao puerpério & 63 & 43,8 \\
Não evitáveis & 17 & 11,8 \\
Ignorado/inconclusivo & 09 & 6,3 \\
\hline
\end{tabular}

Fonte: Secretaria de Saúde do Recife

\section{Discussão}

Os resultados do presente estudo evidenciam uma elevada Razão de Mortalidade Materna (RMM) na cidade do Recife, em torno de 75 por 100.000 nascidos vivos, muito acima das recomendações da OMS, que considera aceitável uma RMM abaixo de 20 por 100.000 nascidos vivos ${ }^{4}$. Observase ainda freqüência alta de sub-registro (aproximadamente $28 \%$ ) nas declarações de óbito, implicando na constatação de que se apenas as informações oficiais fossem utilizadas, quase um terço das mortes maternas não seriam identificadas.

A RMM encontrada para a cidade do Recife encontra-se acima da RMM brasileira não corrigida (em torno de 50), porém abaixo da RMM resultante da aplicação do fator de correção de 2,2 proposto por Laurenti ${ }^{7,8}$. Conforme já foi apontado por Albuquerque et al. ${ }^{10}$, isso ocorre devido a características próprias da cidade do Recife, que representa um grande conglomerado urbano, diferindo das áreas rurais onde, além do preenchimento incorreto das declarações de óbito, a subenumeração é aumentada em decorrência das mortes sem assistência médica e das mortes não 
declaradas (sem atestado de óbito), com sepultamentos em cemitérios clandestinos. No Recife, em função da organização do sistema de saúde, esses últimos problemas não ocorrem e o sub-registro fica, portanto, limitado às mortes não declaradas como maternas nos atestados de óbito.

Destacamos outrossim que, apesar de encontrarmos uma RMM bastante próxima daquela relatada por Albuquerque et al. no Recife em 19921993 (77,7 por 100.000 nascidos vivos), o percentual de subenumeração vem se reduzindo progressivamente. No estudo de Albuquerque et al. esse percentual foi de $52,4 \%{ }^{10}$, ao passo que em nosso estudo foi de $27,8 \%$, assemelhando-se à taxa de sub-registro de países europeus, como a Holanda (estimado em $26 \%)^{11}$. Vale ressaltar que a subenumeração foi ainda consideravelmente menor (em torno de 17\%) nos anos de 1999 e 2000), coincidindo com a adoção da nova ficha de declaração de óbito adotada em 1999, em que os campos 43, 44 e 49 do bloco VI destinam-se ao registro do estado gestacional ${ }^{12}$. Além disso, a Secretaria $\mathrm{Mu}-$ nicipal de Saúde desenvolve, desde 1994, o sistema de investigação de todos os óbitos femininos em idade fértil, utilizando os critérios de Laurenti ${ }^{7,8}$ para identificação dos óbitos maternos presumiveis, e assim a razão oficial de mortalidade materna é bastante confiável.

Considerando a RMM de outras cidades brasileiras, a mortalidade materna encontrada em Recife é superior à relatada para o Rio de Janeiro e São Paulo, que oscilam em torno de 50 para 100.000 nascidos vivos ${ }^{5,13}$ e à referida por Cecatti et al. para a cidade de Campinas $(45,5$ por 100.000 nascidos vivos $)^{9}$, confirmando as desigualdades existentes entre o Nordeste e o Sudeste do Brasil.

Outro grave problema diz respeito tanto às causas como à evitabilidade dos óbitos. Verificamos nítido predomínio das causas obstétricas diretas, representando ainda 69\% das mortes maternas. A grande maioria dessas mortes poderia ter sido evitada, sobretudo pela assistência adequada ao pré-natal, parto e puerpério.

Encontramos a hipertensão como causa principal de mortalidade materna (19\%), corroborando os achados de Cecatti et al. em estudo realizado no Recife ${ }^{14}$, e coincidindo com a maior parte dos estudos brasileiros ${ }^{5,9,13,14}$. Mesmo em países desenvolvidos, como os Estados Unidos, refere-se que em torno de $18 \%$ das mortes maternas decorram de complicações da hipertensão induzida pela gestação ${ }^{2}$. Essas complicações são representadas por edema agudo de pulmão, eclâmpsia, insuficiência renal aguda, síndrome HELLP, descolamento prematuro de placenta (DPPNI), coagulação intravascular disseminada (CIVD) e embolia pulmonar ${ }^{15}$. Destacamos todavia que grande par- te desses óbitos por hipertensão ocorre em mulheres com mais de 30 anos, multíparas e hipertensas crônicas ${ }^{16}$, condições que podem ser facilmente identificadas no pré-natal, adotandose a terapêutica adequada para minimizar as repercussões sobre o binômio mãe-feto, inclusive o internamento precoce e o parto prematuro terapêutico em centros de referência, além, é claro, do óbvio papel do planejamento familiar, uma vez que parcela considerável dessas gestações nunca deveriam ter ocorrido.

Por outro lado, é lamentável que a hemorragia represente ainda causa tão freqüente de morte materna, uma vez que esta em geral é característica da falta de assistência adequada ao parto e ao pós-parto imediato, sendo mais elevada nos países menos desenvolvidos e mais comum em mulheres pobres e com maior paridade 4 . A assistência ao parto assume um papel extremamente relevante, porquanto a maioria das mortes irá ocorrer no período periparto, quando soem acontecer os eventos cataclísmicos, e é essencial a intervenção de profissionais qualificados e hospitais bem aparelhados para o tratamento das emergências obstétricas. Ressaltamos que as mortes maternas foram mais freqüentes durante o domingo e no período da madrugada, apontando que as dificuldades para se obter assistência adequada podem ser maiores no final de semana e durante a noite. No entanto, essa tendência precisa ser confirmada em estudos mais abrangentes, de série temporal.

A infecção puerperal representou outra causa importante de óbito (11\%), embora menos freqüente em relação ao estudo de Cecatti et al., referindo uma freqüência de $19 \%{ }^{14}$. Nesse sentido, cumpre salientar a importante associação de infecção com operação cesariana, porque quase $60 \%$ dos casos de morte por infecção sucederam-se a cesariana, e esta representa indiscutivelmente o principal fator de risco para infecção pós-parto ${ }^{17}$. Atualmente, segundo dados do SINASC, em torno de $40 \%$ dos partos no Recife são operatórios, de forma que além da proposta de antibioticoterapia profilática em toda cesariana ${ }^{17}$ é essencial reduzir esses índices elevados de parto operatório como forma de prevenção de parcela significativa das mortes maternas.

Ao contrário do que foi descrito por outros autores $^{9}$, a mortalidade materna por abortamentos continua elevada no Recife, resultando em 9\% dos óbitos. Cecatti et al. descreveram taxa semelhante, em torno de $11 \%$, atribuída basicamente aos abortos provocados ${ }^{14}$, o que também foi confirmado em nosso estudo. Acreditamos que essa mortalidade continua elevada por conta da proibição da venda de medicamentos habitualmente utilizados para interrupção da gestação, de forma 
que as mulheres decididas a abortar acabam apelando para métodos invasivos, de alto risco, resultando em complicações como hemorragia e, sobretudo, infecção. A maior taxa de complicações nesses abortamentos provocados por outros métodos em relação ao misoprostol já foi demonstrada em outro estudo realizado no Recife ${ }^{18}$.

Por outro lado, quando se analisam as causas indiretas, observa-se elevada freqüência de cardiopatias $(12,5 \%)$, relacionadas sobretudo à febre reumática (potencialmente preveniveis) e, em sua grande maioria, diagnosticadas previamente à gestação sem que, no entanto, as mulheres recebessem orientação contraceptiva adequada, porquanto muitas jamais deveriam ter engravidado, encontrando-se até mesmo multiparas cardiopatas. Além disso, o pré-natal em geral foi iniciado tardiamente, com número inadequado de consultas, e muitas dessas mulheres deveriam ter sido hospitalizadas durante a gravidez, garantindo a assistência ao parto em hospital terciário. Achado semelhante foi descrito por Cecatti et al. ${ }^{14}$.

O presente estudo vem contribuir para a compreensão da problemática da mortalidade materna em nosso meio, reforçando diversos estudos de base populacional que vêm sendo publicados no Brasil nos últimos anos ${ }^{5,9,13,14}$. Além de dimensionar a magnitude da mortalidade materna, todos esses estudos apontam as causas mais freqüentes em nosso país, que continuam sendo as obstétricas diretas, sobressaindo-se a hipertensão, e destacam aspectos sociodemográficos e de assistência médica que devem ser levados em consideração nos programas de saúde pública, para elaborar estratégias eficazes de prevenção. Evidentemente, a discussão não deve se restringir a esses estudos, uma vez que é necessário aprofundá-la e estudar com atenção os fatores de risco associados ao óbito materno. Entretanto, o mais importante é reconhecer que, caracterizando-se como evitáveis a maioria dos óbitos, todos os esforços devem ser envidados no sentido de desenvolver medidas de saúde pública para melhorar a qualidade de assistência prénatal, ao parto e puerpério e, em uma visão holística, promover a saúde reprodutiva como direito de cidadania.

\section{ABSTRACT}

Purpose: to determine the Maternal Mortality Ratio (MMR) among women living in the city of Recife, Brazil through the analysis of all death certificates of women aged 10-49 years from 1994 to 2000. To determine the underreporting rate and to study the main characteristics, basic causes, classification and avoidance of maternal deaths.

Methods: a descriptive population-based study was conducted and all death certificates of women aged 10-49 years were analyzed and classified as declared or presumed (Laurenti criteria). Clinical records and autopsy data, when available, were studied and basic cause and underreporting rate were determined. Maternal Mortality Ratio was calculated using information on live births from SINASC ("Sistema de Informações dos Nascidos Vivos").

Results: a total of 144 maternal deaths were identified (declared $=104 ;$ presumed and confirmed after investigation=44). The Maternal Mortality Ratio was 75.5 per 100,000 live births. The underreporting rate was $27.8 \%$. A predominance of direct causes was observed (about 69\%) and the most frequent causes of death were hypertension (19\%), hemorrhage (16\%) and infection (11\%). About $82 \%$ of the deaths were considered avoidable by adequate antenatal, delivery and post-partum care.

Conclusions: Maternal Mortality Ratio is high in the city of Recife, Brazil and the underreporting rate is still high. Direct obstetric causes and avoidable deaths are predominant. There is a lack of adequate antenatal, delivery and postpartum care.

KEY WORDS: Maternal death. Reproductive health. Causes of death, Vital statistics.

\section{Referências}

1. Souza ML, Laurenti R. Mortalidade Materna: conceitos e aspectos estatísticos. Centro da OMS para a Classificação das Doenças em Português (MS/ USP/ OPAS/ OMS). São Paulo: Série Divulgação; 1987.

2. Koonin LM, MacKay AP, Berg CJ, Atrash HK, Smith JC. Pregnancy-related mortality surveillanceUnited States, 1987-1990. MMWR CDC Surveillance Summaries 1997; 46: 17-36.

3. CID-10. Classificação Estatística Internacional de Doenças e Problemas Relacionados à Saúde. 10 Revisão. Organização Mundial de Saúde. Centro Colaborador da OMS para a Classificação das Doenças em Português. São Paulo: EDUSP; 1997.

4. WHO/UNICEF (World Health Organization/ United Nations International Children's Emergency Fund), 1996. Revised 1990 estimates of Maternal Mortality. A new approach by WHO and UNICEF. WHO/FRH/ MSM 96.11. UNICEF/PZN/96.1. Geneva: WHO/ UNICEF.

5. Haddad N, Silva MB. Mortalidade feminina em idade reprodutiva no estado de São Paulo, Brasil, 19911995: causas básicas de óbito e mortalidade materna. Rev Saúde Pública 2000; 34: 64-70. 
6. Ministério da Saúde. Mortalidade Brasil - 1995. Brasília, Fundação Nacional de Saúde, 1998.

7. Laurenti R. Marcos referenciais para estudos e investigações em Mortalidade Materna. Rev Saúde Pública, São Paulo, 1988; 22: 507-12.

8. Laurenti R, Jorge MHPM, Gotlieb SL. Reflexões sobre a mensuração da mortalidade materna. Cad Saúde Pública 2000; 16: 23-30.

9. Cecatti JG, Faúndes A, Surita FGC. Maternal mortality in Campinas: Evolution, underregistration and avoidance. Rev Paul Med 1999; 117: 5-12.

10.Albuquerque RM, Cecatti JG, Hardy E, Faúndes A. Mortalidade Materna em Recife. Avaliação da subenumeração dos registros oficiais. Cad Saúde Públ 1997; 13: 59-65.

11.Schuitemaker N, Van Roosmalen J, Dekker G, Van Dongen P, Van Geijn H, Gravenhorst JB. Underreporting of maternal mortality in The Netherlands. Obstet Gynecol 1997; 90: 78-82.

12.Laurenti R, Jorge MHPM, Gotlieb SL. Mortes maternas no Brasil: análise no preenchimento de variável da declaração de óbito. Informe Epidemiológico do SUS 2000; 9: 43-50.
13.Theme-Filha MM, Silva RI, Noronha CP. Mortalidade Materna no município do Rio de Janeiro, 1993 a 1996. Cad Saúde Pública 1999; 15: 397-403.

14.Cecatti JG, Albuquerque RM, Hardy E, Faúndes A. Mortalidade Materna em Recife. Causas de óbitos maternos. Rev Bras Ginecol Obstet 1998; 20: 7-11.

15. Onrust S, Santerna JG, Aarnoudse JG. Preeclampsia and the HELLP syndrome still cause maternal mortality in The Netherlands and other developed countries; can we reduce it? Eur J Obstet Gynecol Reprod Biol 1999; 82:1, 41-46.

16.Amorim MMR, Santos LC, Katz L. Fatores de risco para morte materna na pré-eclâmpsia-eclâmpsia. Braz J Mother Child Health (in press).

17.Smaill F, Hofmeyr GJ. Antibiotic prophylaxis for cesarean section. Cochrane Database Syst Rev 2000; (2): CD000933.

18.Faundes A, Santos LC, Carvalho M, Gras C. Postabortion complications after interruption of pregnancy with misoprostol. Adv Contracept 1996; 12:1-9.

\title{
II Congresso de Ginecologia e Obstetrícia da Região Norte
}

\section{3 a 26 de outubro de 2002}

\author{
Porto Velho - RO
}

\author{
Informações: \\ SOGIRO \\ (69) 224-1974 \\ sogiro@enter-net.com.br
}

